\title{
The effect of substitution and isomeric imperfection on the photophysical behaviour of $p$-phenylenevinylene trimers
}

\author{
J. Seixas de Melo ${ }^{\text {a,* }}$, J. Pina ${ }^{\text {a }}$, H.D. Burrows ${ }^{a, *}$, S. Brocke ${ }^{b}$, \\ O. Herzog ${ }^{\mathrm{b}}$, E. Thorn-Csányi ${ }^{\mathrm{b}}$ \\ a Chemistry Department, University of Coimbra, Rua Larga, P3004-535 Coimbra, Portugal \\ ${ }^{\mathrm{b}}$ Institut für Technische und Makromolekulare Chemie, Universität Hamburg, Bundesstraße 45, D-20146 Hamburg, Germany
}

Received 29 December 2003; in final form 24 February 2004

Published online: 24 March 2004

\begin{abstract}
Spectroscopic and photophysical properties of two $p$-phenylenevinylene (PV) trimers, 2,5-substituted diheptyl-( $p$-phenylenevinylene) and di-[(2-ethylhexyl)oxy]-( $p$-phenylenevinylene), were studied using absorption spectroscopy, fluorescence and laser flash photolysis. The change from alkyl to alkyloxy groups red-shifts the absorption and fluorescence bands. The rate of internal conversion is independent of the substitution, whereas alkyloxy substitution increases the $S_{1} \leadsto T_{1}$ intersystem crossing rate by an order of magnitude. The relevance for the behaviour of conjugated PPV polymers is discussed. For diheptyl-PV, a sample having ca. 3\% of the cis-configuration was also studied. Comparison between the all-trans and the cis-contaminated samples revealed no significant differences in their photophysical properties.
\end{abstract}

(C) 2004 Elsevier B.V. All rights reserved.

\section{Introduction}

Within the last decade, conjugated organic polymers have reached the role of major, technologically important class of materials. Conjugated organic polymers have the advantages of low cost, ease of modification of properties by appropriate substitution and of solvent processability. One major application is as light emitting display devices, which are finding use for the next generation of mobile phones [1]. Of relevance to this are their photophysical properties, which are complex, with many different excited states [2] and other transient species observed over various time domains [3,4]. This complexity is also influenced by their many possible conformational states [2] and chemical imperfections [5-7].

For practical applications there is however one important limiting theoretical restraint on both their electroluminescence yields, and long term stability: the formation of triplet states. Usually, only the excited

\footnotetext{
${ }^{*}$ Corresponding authors. Fax: +351-239-827703.

E-mail addresses: sseixas@ci.uc.pt (J. Seixas de Melo), burrows@ ci.uc.pt (H.D. Burrows).
}

singlet states are luminescent, although there is now evidence of phosphorescence in the ladder poly(paraphenylene) polymer [8], and it is generally accepted that triplet state formation should be considered as the major causes of efficiency loss in these systems. Triplet states are also involved in the photooxidative degradation of these systems $[9,10]$.

The complexity of the polymeric systems is frequently overcome by performing the study of oligomeric analogues $[7,11,12]$ and it has been argued that with a number of unimer units superior [13] or equal to five $[11,12,14,15]$, in the case of the polythiophenes, 15 in the case of the poly-p-phenylenevinylene [16] and 12 in the case of polyfluorene [17], the spectroscopic properties of the oligomers become equivalent to those of the corresponding polymers. The oligomer approach is therefore a useful guide to the study of polymeric defects resulting from chemical imperfections. Within these, conjugation interruptions, degradation resulting from oxidation of the backbone chain and contaminations resulting from the synthesis are generally among the major important factors to be considered as potential inhibitors for a perfect LED behaviour. 
A conjugated polymer family of particular importance is the poly( $p$-phenylenevinylene)s (PPV). The first observation of luminescence in a fully conjugated polymer involved unsubstituted PPV [18]. This is still an important material for light emitting devices and other applications. Unfortunately, it is insoluble in common solvents, and films are normally prepared by thermal decomposition of soluble precursors. In contrast, derivatives, such as the 2-methoxy-5-(2'-ethylhexyloxy) polymer MEH-PPV [19], are solvent processable, and also show good electroluminescence. However, differences are observed in both the spectroscopic properties [20] and photochemical stability between unsubstituted (or alkyl substituted) PPVs and the alkyloxy substituted derivatives.

The present work aims to clarify origin of the differences in photophysics between these derivatives by carrying out a comprehensive photophysical study of two, fully-trans-p-phenylenevinylene trimers, having alkyl and alkyloxy substituents in the 2,5-positions, focusing on the determination of the complete set of rate constants for $S_{1}$ deactivation and the efficiency of triplet state formation. It is important to compare derivatives with the same substitution pattern, since theoretical calculations show that the 2,5-derivatives have little effect on the transition energies relative to PPV, but increase the oscillator strength of the lowest energy transitions by destroying both the chargeconjugation symmetry and spatial symmetry [20]. In addition, with the alkyl derivative, the effect of isomeric contamination, resulting from a small percentage of the cis trimer on the photophysical properties was observed.

\section{Experimental}

Solvents were of spectroscopic or equivalent grade. Concentrations of solutions were in the range from $10^{-6}$ to $1 \times 10^{-5} \mathrm{M}$. These were deoxygenated by bubbling with nitrogen or argon. Methylcyclohexane was purified by the procedure described in [21].

Absorption and luminescence spectra were recorded on Shimadzu UV-2100, Olis-Cary 14, and Jobin-Ivon SPEX Fluorolog 3-22 spectrometers, respectively. Fluorescence spectra were corrected for the wavelength response of the system. Special care was taken with the slit widths of both excitation and emission monochromators, which were kept $\leqslant 0.5 \mathrm{~mm}$.

Fluorescence quantum yields were measured using $\alpha$-terthienyl in ethanol as standard $\left(\phi_{\mathrm{F}}=0.054\right)$ [11]. The fluorescence quantum yields at $77 \mathrm{~K}$ were obtained by comparison of the spectra at this temperature and $293 \mathrm{~K}$ run under the same experimental conditions and the $\phi_{\mathrm{F}}$ value was calculated assuming that the sample volume ratio $V_{293 \mathrm{~K}} / V_{77 \mathrm{~K}}=0.8[22]$.
The molar extinction coefficients $(\varepsilon)$ were obtained at absorption maxima from the slope of the plot of the absorption vs. the concentration using seven solutions of different concentrations (correlation values $\geqslant 0.999$ ).

Triplet-singlet difference absorption spectra and yields were obtained using an Applied Photophysics laser flash photolysis equipment pumped by the third harmonic of a Nd:YAG laser (Spectra Physics) with excitation wavelength of $355 \mathrm{~nm}$ [14]. First-order kinetics was observed for the decay of the lowest triplet state. The transient spectra $(300-650 \mathrm{~nm})$ were obtained by monitoring the optical density change at $5-10 \mathrm{~nm}$ intervals, averaging at least 10 decays at each wavelength.

In all cases the signal was assigned to a triplet state because:

(i) it was quenched by oxygen (quenching constant by oxygen, $k_{\mathrm{ox}}=2.5-16 \times 10^{9} \mathrm{M}^{-1} \mathrm{~s}^{-1}$ ):

(ii) it decayed by first-order kinetics with microsecond lifetimes;

(iii) other possible transients, such as radical ions are generally not produced on photolysis in the nonpolar solvent benzene.

The triplet molar absorption coefficients obtained in methylcyclohexane were determined by the singlet depletion technique, according to the well-known relationship [22]

$\varepsilon_{\mathrm{T}}=\frac{\varepsilon_{\mathrm{S}} \times \Delta \mathrm{OD}_{\mathrm{T}}}{\Delta \mathrm{OD}_{\mathrm{S}}}$,

where both $\Delta \mathrm{OD}_{\mathrm{S}}$ and $\Delta \mathrm{OD}_{\mathrm{T}}$ are obtained from the triplet-singlet difference transient absorption spectra. The $\phi_{\mathrm{T}}$ values were obtained by comparing the $\Delta \mathrm{OD}$ at $525 \mathrm{~nm}$ of a benzene solution of benzophenone (the standard) and of the compound (optically matched at the laser wavelength) using the equation [22]

$\phi_{\mathrm{T}}^{\text {Compound }}=\frac{\varepsilon_{\mathrm{TT}}^{\text {Benzophenone }}}{\varepsilon_{\mathrm{TT}}^{\text {Compound }}} \cdot \frac{\Delta \mathrm{OD}_{\max }^{\text {Compound }}}{\Delta \mathrm{OD}_{\max }^{\text {Benzophenone }}} \cdot \phi_{\mathrm{T}}^{\text {Benzophenone }}$.

Fluorescence decays were measured using a homebuilt Time-Correlated Single Photon Counting apparatus with an $\mathrm{N}_{2}$ filled IBH 5000 coaxial flashlamp as excitation source, Jobin-Ivon monochromator, Philips XP2020Q photomultiplier, and Canberra instruments Time-to-amplitude converter and Multichannel Analyser. Alternate measurements (1000 counts per cycle), controlled by Decay ${ }^{\circledR}$ software (Biodinâmica-Portugal), of the pulse profile at 337 or $356 \mathrm{~nm}$ and the sample emission were performed until $1-2 \times 10^{4}$ counts at the maximum were reached [23]. The fluorescence decays were analysed using the modulating functions method of Striker with automatic correction for the photomultiplier 'wavelength shift' [24]. 


\section{Results and discussion}

The structures and abbreviations of the studied samples are depicted in Fig. 1. They consist in 2,5substituted $p$-phenylenevinylene derivatives possessing identical backbone structures but differing in the substitution promoted by the side chain, and are here abbreviated as HPV3 (all-trans-2,5-diheptyl-( $p$-phenylenevinylene) trimer) and EHOPV3 (all-trans-2,5-di-(2'ethylhexyloxy)-( $p$-phenylenevinylene) trimer). In addition, the photophysical properties of a sample of HPV3 with $3 \%$ of the corresponding cis isomer (HPV3/cis) were observed.

The absorption spectra of the three samples are shown in Fig. 2. The substitution of an alkyl group by an alkyloxy group red-shifts the absorption maximum of the compound by ca. $69 \mathrm{~nm}$ (ca. $0.55 \mathrm{eV}$ ). Similar shifts have previously been reported between the polymer PPV and the 2,5-alkyloxy derivative MEHPPV [25], and have been interpreted theoretically through ZIN$\mathrm{DO} / \mathrm{S}$-level [26] calculations in terms of the antibonding interaction with the oxygen $\pi$-orbitals. Recent investigations with analogous oligomers, but with $n$ ranging from $n=1$ to 7 , have shown a linear increase in the

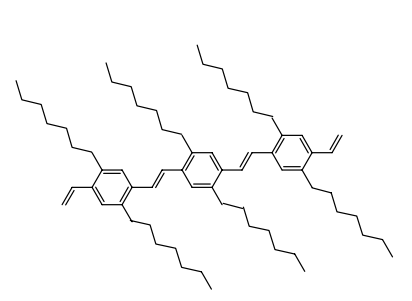

HPV3 all-trans

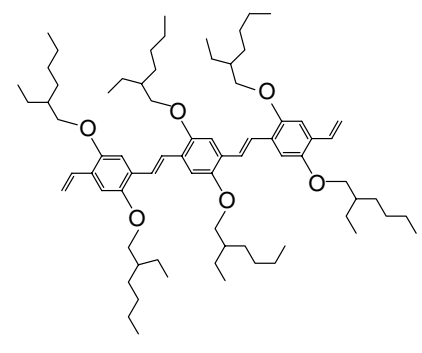

EHOPV3 all-trans
Fig. 1. Structural formula of PV oligomers investigated with the acronyms utilized in the text: 2,5-diheptyl $p$-phenylenevinylene trimer (HPV3); 2,5-di-[(2-ethylhexyl)-oxy] $p$-phenylenevinylene trimer (EHOPV3).

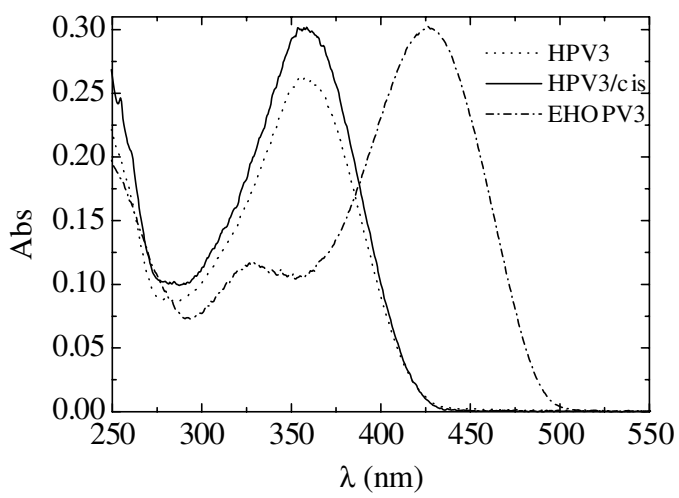

Fig. 2. Absorption spectra of HPV3, HPV3/cis and EHOPV3 in methylcyclohexane at $T=293 \mathrm{~K}$. energy of the $\mathrm{S}_{0} \rightarrow \mathrm{S}_{1}$ electronic transition with $n$ [27,28]. The spectroscopic study there presented was mainly restricted to optical (absorption and steady state fluorescence) properties of the pure all-trans oligomers at room and low temperatures, including site-selective fluorescence measurements $[27,28]$. These studies also provided indications of aggregation of the oligomers in solution on increasing concentration.

Comparing the $\mathrm{S}_{0} \rightarrow \mathrm{S}_{1}$ spectra for HPV3 and HPV3/ cis, only a minor change in the absorption maximum of $\approx 1 \mathrm{~nm}$ is observed.

Fluorescence spectra and quantum yields were determined for the compounds at room temperature and $77 \mathrm{~K}$. The fluorescence spectra of samples HPV3 and $\mathrm{HPV} 3 / \mathrm{cis}$ at room temperature are fairly similar; see Fig. 3 and Table 1. However, the shift in emission maximum of HPV3 relative to EHOPPV3 is now $\approx 48$ $\mathrm{nm}(0.3 \mathrm{eV})$, substantially less than that found in absorption. Note that in the case of HPV3 there is a blue shift of the emission band when going to low temperature, and also at $77 \mathrm{~K}$ the emission spectra is now devoid of vibrational resolution. Also at $77 \mathrm{~K}$, the emission maximum for compound HPV3/cis (with 3\% cis impurity) is blue shifted about $3 \mathrm{~nm}$ relative to the all-trans HPV3. For HPV3 and HPV3/cis, there is no change in the fluorescence quantum yield from $293 \mathrm{~K}$ to $77 \mathrm{~K}$, see Table 2, indicating that the excited state $S_{1}$ most probably has the same geometry at both temperatures. Two possible explanations can be given for the observed blue shift, and loss of structure in the emission spectra of HPV3 on cooling. One concerns the geometry of the ground-state to which $S_{1}$ deactivates, and may be associated with the existence of a greater population of lower energy planar conformers at low temperature. It is now well-established that conjugated organic polymers, and oligomers, tend to possess a quinoidal-type structure in their $S_{1}$ state $[11,14]$. The emission at $77 \mathrm{~K}$ would then be from an $\mathrm{S}_{1}$ state with the same conformation (and energy) as at $293 \mathrm{~K}$, but to a 'more planar', i.e. lower energy, ground state. As a consequence, the en-

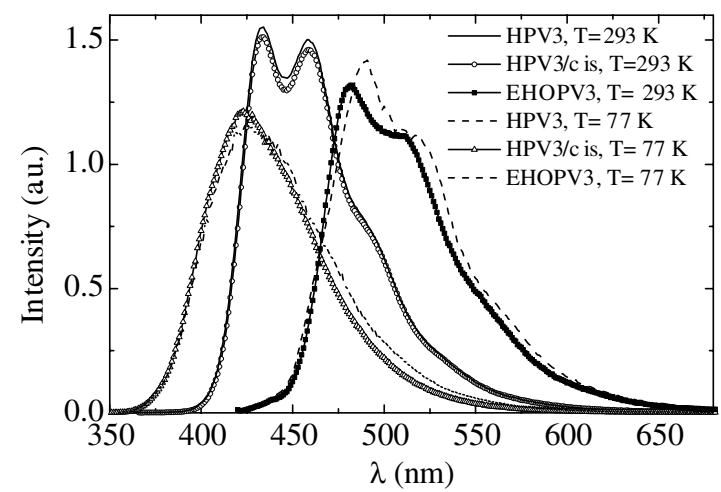

Fig. 3. Emission spectra of HPV3, HPV3/cis and EHOPV3 in methylcyclohexane at room $(293 \mathrm{~K})$ and low $(77 \mathrm{~K})$ temperatures. 
Table 1

Spectroscopic Properties of HPV3, HPV2/cis and EHOPV3 in methylcyclohexane

\begin{tabular}{lllllll}
\hline Compound & $\begin{array}{l}\lambda_{\max }^{\text {abs }} \\
(\mathrm{nm})\end{array}$ & $\begin{array}{l}\varepsilon_{\max }^{\mathrm{S}_{0} \mathrm{~S}_{1}} \\
\left(\mathrm{M}^{-1} \mathrm{~cm}^{-1}\right)\end{array}$ & $\begin{array}{l}\lambda_{\max }^{\mathrm{m} \mathrm{b}} \\
(293 \mathrm{~K})\end{array}$ & $\begin{array}{l}\lambda_{\max }^{\mathrm{em}} \\
(77 \mathrm{~K})\end{array}$ & $\begin{array}{l}\lambda_{\max }^{\mathrm{T}_{1} \rightarrow \mathrm{T} n} \\
(\mathrm{~nm})\end{array}$ & $\begin{array}{l}\varepsilon_{\max }^{\mathrm{T}_{1} \rightarrow \mathrm{T}} \\
\left(\mathrm{M}^{-1} \mathrm{~cm}^{-1}\right)\end{array}$ \\
\hline HPV3 & 360 & 52200 & 434,458 & 426 & 565 & 195600 \\
HPV3/cis & 359 & $(\mathrm{a})$ & 434,459 & 423 & 565 & 179600 \\
EHOPV3 & 427 & 63000 & 482 & 490 & 560 & 122940 \\
MEHPPV $^{\mathrm{a}}$ & 501 & $1.33 \times 10^{8}$ & 554,597 & - & 830 & - \\
\hline
\end{tabular}

For the polymer MEHPPV data is in benzene.

(a) not determined.

${ }^{\text {a }}$ Data from [9] in benzene.

${ }^{\mathrm{b}}$ In cases where more than one value exists, the underlined is the maximum.

Table 2

Photophysical properties of HPV3, HPV3/cis and EHOPV3 in methylcyclohexane

\begin{tabular}{|c|c|c|c|c|c|c|c|c|c|c|c|}
\hline Compound & $\begin{array}{l}\phi_{\mathrm{F}} \\
(293 \mathrm{~K})\end{array}$ & $\begin{array}{l}\phi_{\mathrm{F}} \\
(77 \mathrm{~K})\end{array}$ & $\begin{array}{l}\tau_{\mathrm{F}} \\
(\mathrm{ns})\end{array}$ & $\phi_{\mathrm{T}}$ & $\begin{array}{l}\tau_{\mathrm{T}} \\
(\mu \mathrm{s})\end{array}$ & $\begin{array}{l}k_{\mathrm{F}}\left(\phi_{\mathrm{F}} / \tau_{\mathrm{F}}\right) \\
\left(\mathrm{ns}^{-1}\right)\end{array}$ & $\begin{array}{l}k_{\mathrm{NR}} \\
\left(\mathrm{ns}^{-1}\right)\end{array}$ & $\phi_{\mathrm{IC}}$ & $\begin{array}{l}k_{\mathrm{IC}} \\
\left(\mathrm{ns}^{-1}\right)\end{array}$ & $\begin{array}{l}k_{\mathrm{ISC}} \\
\left(\mathrm{ns}^{-1}\right)\end{array}$ & $\begin{array}{l}k_{\mathrm{ox}} \\
\left(10^{9} \mathrm{M}^{-1} \mathrm{~s}^{-1}\right)\end{array}$ \\
\hline HPV3 & 0.64 & 0.60 & 1.40 & 0.005 & 3.9 & 0.46 & 0.26 & 0.355 & 0.254 & 0.0036 & 2.5 \\
\hline $\mathrm{HPV} 3 /$ cis & 0.65 & 0.62 & 1.32 & $0.006^{\mathrm{b}}$ & 4.0 & 0.49 & 0.51 & 0.344 & 0.26 & 0.005 & 3.3 \\
\hline EHOPV3 & 0.61 & 0.44 & 1.72 & 0.025 & 94 & 0.36 & 0.23 & 0.365 & 0.21 & 0.015 & 16 \\
\hline MEHPPV $^{a}$ & 0.28 & - & 0.3 & $0.012_{5}$ & 92 & 0.93 & 2.4 & 0.75 & 2.5 & 0.042 & - \\
\hline
\end{tabular}

For the polymer MEHPPV data is in benzene.

${ }^{\text {a }}$ Data from [9] in benzene.

${ }^{\mathrm{b}}$ The $\phi_{\mathrm{T}}$ value was obtained assuming the $\varepsilon_{\max }$ for HPV3 (see Table 1).

ergy difference between the $0-0$ levels of $\mathrm{S}_{0}$ and $\mathrm{S}_{1}(\Delta E)$ at $77 \mathrm{~K}$ would be greater than at $293 \mathrm{~K}$, leading to the observed blue shift in the fluorescence for HPV3 (and HPV3/cis). A similar pattern has been found for the oligomers of thiophene [14] and mixed thiophene-pyrrol with alkyl side-chains [29]. Low temperature absorption spectra show a red-shift of the maxima $(\approx 8 \mathrm{~nm})$ and an overlap with the high energy part of the emission band, and it is likely that loss in vibronic structure is due to self-absorption. An alternative explanation in terms of aggregation is less likely since this has been shown to lead to a large shift of luminescence in related systems [28].

For the EHOPV 3 case, the behaviour is the opposite of that found for HPV3, and there is now a red-shift of $\approx 8 \mathrm{~nm}$ of the fluorescence maximum when going from RT to $77 \mathrm{~K}$, and no loss of vibronic structure. In the case of EHOPV3, spectra are independent of concentration indicating that self-absorption is not important. This difference between the two derivatives may lie in the structural modification induced by the alkyloxy vs. alkyl side-chains. As suggested by theoretical calculations [26], the electron-donor alkyloxy group will extend the $p$ - $\pi$ electrons of the oxygen atoms to the $p$-phenylenvinylene backbone. As a consequence of this, the absorption and emission, wavelength maxima are significantly lower in energy. The absence of differences in the emission spectra on going from $293 \mathrm{~K}$ to $77 \mathrm{~K}$ indicates that no severe structural changes have been induced. Only a small red-shift between $293 \mathrm{~K}$ and $77 \mathrm{~K}$ is observed for EHOPV3 $(\approx 8 \mathrm{~nm})$ which again supports the absence of significant conformational change or aggregation on going to low temperature. Comparing the emission spectra changes with temperature of HPV3 with the lack of effect for EHOPV3, and absorption spectral behaviour, it appears that the oxygen atom may act as an electron donor to the conjugated polymer $\pi$-electron backbone. More detailed studies on emission spectra in a series of alkyloxyl-PPV derivatives have been given in references [27,28,30,31].

The triplet states of the three trimers were studied by laser flash photolysis. Here, it was found, however, that the observed pattern in the singlet properties does not have a parallel in the triplet-singlet absorption spectra. In fact, the triplet absorption maximum for EHOPV3 is blue-shifted by ca. $5 \mathrm{~nm}$ when compared with the value for HPV3, see Table 1 and Fig. 4. Although these spectra are strictly triplet-singlet difference spectra, the changes occur in a region where there is no ground state absorption, and consequently do reflect genuine differences. This would suggest that the triplet state in EHOPV3 is less delocalized than in HPV3, in contrast to the behaviour found in the singlet state. A decrease in the $\mathrm{S}_{1}-\mathrm{S}_{0}$ gap, i.e., in the singlet energy transition, as the conjugation length increases is generally accepted to imply that increased conjugation length delocalises excitations on the oligomer or polymer backbone [32]. Evidence for this can be found in experiments where model compounds [33,34] have shown a general trend translated by empirical equations for oligomers that are dependent on the actual $S_{1}-S_{0}$ energy difference and on the predicted chain length. Since the triplet states can essentially be considered as consisting of the same orbitals as the singlets, they should show a similar 

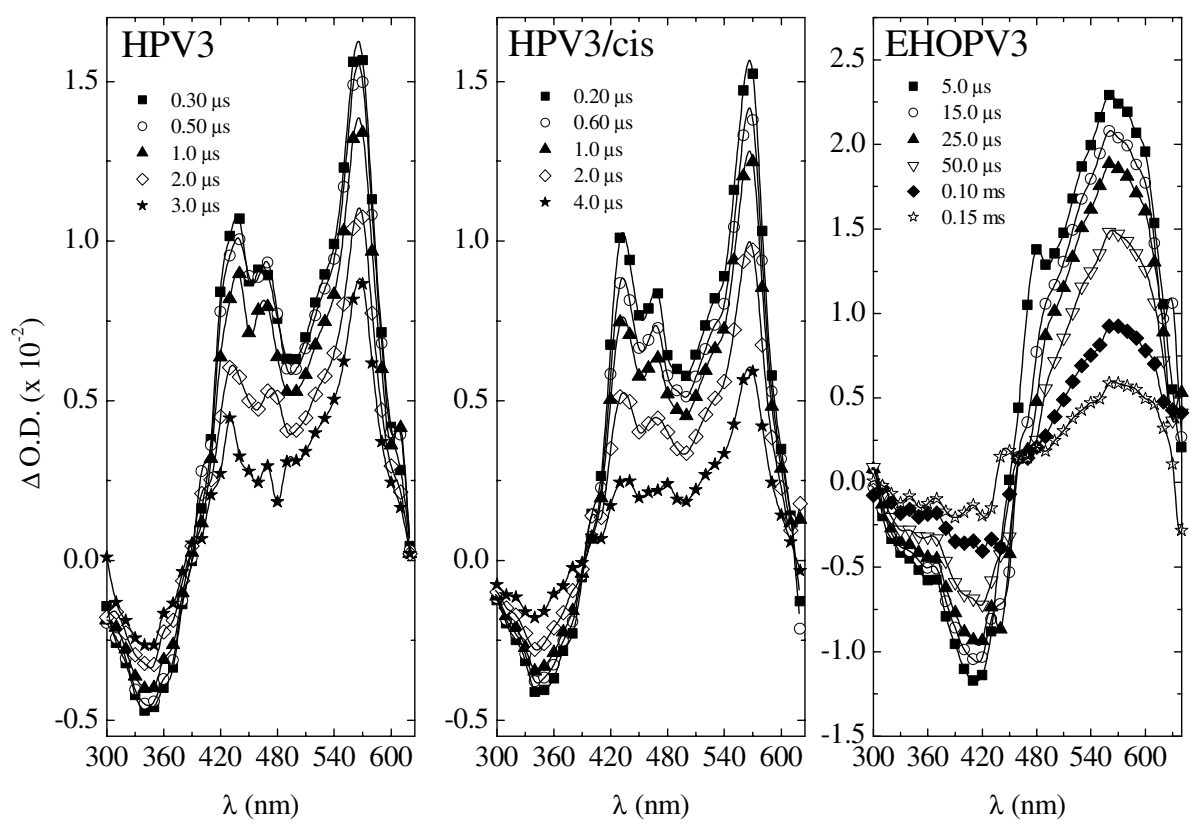

Fig. 4. Transient triplet-triplet absorption spectra of HPV3, HPV3/cis and EHOPV3 in methylcyclohexane at $T=293 \mathrm{~K}$.

dependence, i.e., increased delocalization with the increased conjugation length [35]. It has been observed in a broad range of conjugated polymers that the triplet gap $\left(\mathrm{T}_{1}-\mathrm{T}_{n}\right)$ decreases as the singlet gap $\left(\mathrm{S}_{0}-\mathrm{S}_{1}\right)$ decreases (in this case the delocalization increases) [35]. As a consequence of this, in conjugated polymers, the triplet states must be more tightly bound (more localized) than the singlet states. However, their degree of localization is strongly affected by the chain backbone conjugation. In the present case with the oligomers, the effect of the alkyloxy chain inducing less delocalization of the triplet state may imply a more localized triplet state in EHOPV3.

From the flash photolysis experiments it was possible to obtain triplet quantum yields $\left(\phi_{\mathrm{T}}\right)$, which allow the determination of all the relevant photophysical properties of the trimers (Table 2). The introduction of the alkyloxy groups decreases the absolute value of the fluorescence quantum yield, increasing both the internal conversion and intersystem crossing quantum yields. However, the most important feature in the comparative photophysics of compounds HPV3 and EHOPV3, is the increase, by an order of magnitude, of the $S_{1} \rightsquigarrow T_{1}$ intersystem crossing rate constant, while the $k_{\mathrm{F}}$ and $k_{\mathrm{IC}}$ rate constants remain constant. In agreement with the spectroscopic data obtained from absorption and fluorescence, we believe that the oxygen atoms have $p-\pi$ electrons that are able to delocalize within the main backbone of the compound. Moreover, it is probable that most of the differences in the photophysics of HPV3 and EHOPV3 result from the increase in the spin-orbit coupling due to the oxygen atom attached to the phenyl ring (spin-orbit coupling constants $\xi=32 \mathrm{~cm}^{-1}$ (C); 154
$\mathrm{cm}^{-1}(\mathrm{O})$; [22]). The bigger intersystem crossing yields in the alkyloxy PPV derivatives compared with the alkyl ones provides a reasonable explanation for the lower photostability of the former compounds. One of the main photodegradation pathways of these systems involves reaction of singlet oxygen, which is formed by energy transfer from the corresponding oligomer or polymer $[9,10]$. The alkyloxy derivatives, having higher quantum yields for triplet formation will, thus, sensitise the production of more singlet oxygen, leading to more photooxidation.

For LED applications of the oligomers and polymers, the relative importance of radiative and nonradiative deactivation of excited states is an important factor for device efficiency, and it is worthwhile comparing the trimer properties with those of the analogous polymers, in this case MEHPPV [9]. From the comparative data for solutions, presented in Table 2, it can be seen that with the polymer the value of $\phi_{\text {IC }}$ increases at the expense of both $\phi_{\mathrm{F}}$ and $\phi_{\mathrm{ISC}}$, and is the main deactivation pathway of the lowest excited singlet state. Although intersystem crossing quantum yields have estimated errors of $\approx 15-20 \%$, with MEHPPV, identical experimental $\phi_{\mathrm{T}}$ values have been established by two independent techniques [9]. In addition, with the analogous alkyloxyPPV oligomers, Candeias et al. [16] have determined values of 0.06 for $\phi_{\mathrm{T}}$, which are close to our 0.08 value for EHOPV3 (Table 2). We, therefore, have considerable confidence in values given in Table 2 . The importance of nonradiative pathways in the decay of the excited singlet states of polythiophenes has recently been confirmed [15]. In this case, the $S_{1} \leadsto S_{0}$ internal conversion process is the dominant deactivation process in 
the polymers [15], in contrast to what has been found with the oligomers [11]. Although care is needed in extrapolating excited state processes from one system to another, the present results seem to indicate a pattern where the relative contribution of the internal conversion channel increases in conjugated polymers when compared to their corresponding oligomers.

\section{Conclusions}

The effect of substitution and of isomeric contamination has been investigated with two 2,5-substituted $p$-phenylenevinylene trimers. It was found that the change in substitution from an alkyl to an alkoxy group results in increase of the $\pi$-electron delocalization in the main backbone chain as a consequence of the electron donating ability of the oxygen atoms. This substitution induces higher triplet yield value as a result of increased spin-orbit coupling. Although the effect on overall luminescence yields of devices is likely to be relatively modest, it may have important consequences on the stability. The contamination of the all-trans alkyl $p$-phenylenevinylene trimer, with a small percentage of the cis conformer, does not seem to influence the general pattern for the photophysical data, suggesting that such levels of isomeric impurities will not have a major effect on device efficiencies.

\section{Acknowledgements}

Financial support from PRAXIS XXI, POCTI, FCT and FEDER is gratefully acknowledged.

\section{References}

[1] R.H. Friend, R.W. Gymer, A.B. Holmes, J.H. Burroughes, R.N. Marks, C. Taliani, D.D.C. Bradley, D.A. Dos Santos, J.L. Bredas, M. Logdlund, W.R. Salaneck, Nature 397 (1999) 121.

[2] F.B. Dias, A. Macanita, J. Seixas de Melo, H.D. Burrows, R. Güntner, U. Scherf, A.P. Monkman, J. Chem. Phys. 118 (2003) 7119 .

[3] N.T. Harrison, G.R. Hayes, R.T. Phillips, R.H. Friend, Phys. Rev. Lett. 77 (1996) 1881.

[4] S.V. Frolov, W. Gellermann, M. Ozaki, K. Yoshino, Z.V. Vardeny, Phys. Rev. Lett. 78 (1997) 729.

[5] L.P. Candeias, G. Padmanaban, S. Ramakrishnan, Chem. Phys. Lett. 349 (2001) 394.

[6] E.J.W. List, R. Guentner, P.S. de Freitas, U. Scherf, Adv. Mater. 14 (2002) 374.
[7] E.J.W. List, J. Partee, J. Shinar, U. Scherf, K. Mullen, E. Zojer, K. Petritsch, G. Leising, W. Graupner, Phys. Rev. B 61 (2000) 10807.

[8] Y.V. Romanovskii, A. Gerhard, B. Schweitzer, U. Scherf, R.I. Personov, H. Bassler, Phys. Rev. Lett. 84 (2000) 1027.

[9] H.D. Burrows, J. Seixas de Melo, C. Serpa, L.G. Arnaut, A.P. Monkman, I. Hamblett, S. Navaratnam, J. Chem. Phys. 115 (2001) 9601.

[10] R.D. Scurlock, B.J. Wang, P.R. Ogilby, J.R. Sheats, R.L. Clough, J. Am. Chem. Soc. 117 (1995) 10194.

[11] R.S. Becker, J. Seixas de Melo, A.L. Macanita, F. Elisei, J. Phys. Chem. 100 (1996) 18683

[12] J. Seixas de Melo, L.M. Silva, L.G. Arnaut, R.S. Becker, J. Chem. Phys. 111 (1999) 5427.

[13] M.G. Harrison, D. Fichou, F. Garnier, A. Yassar, Opt. Mater. 9 (1998) 53.

[14] J. Seixas de Melo, L.M. Silva, M. Kuroda, J. Chem. Phys. 115 (2001) 5625.

[15] J. Seixas de Melo, H.D. Burrows, M. Svensson, M.R. Andersson, A.P. Monkman, J. Chem. Phys. 118 (2003) 1550.

[16] L.P. Candeias, J. Wildeman, G. Hadziioannou, J.M. Warman, J. Phys. Chem. B 104 (2000) 8366.

[17] G. Klaerner, R.D. Miller, Macromolecules 31 (1998) 2007.

[18] J.H. Burroughes, D.D.C. Bradley, A.R. Brown, R.N. Marks, K. Mackay, R.H. Friend, P.L. Burns, A.B. Holmes, Nature 347 (1990) 539.

[19] D. Braun, A.J. Heeger, Appl. Phys. Lett. 58 (1991) 1982.

[20] M. Chandross, S. Mazumdar, M. Liess, P.A. Lane, Z.V. Vardeny, M. Hamaguchi, K. Yoshino, Phys. Rev. B 55 (1997) 1486.

[21] J. Seixas de Melo, R.S. Becker, F. Elisei, A.L. Macanita, J. Chem. Phys. 107 (1997) 6062.

[22] S. Murov, I. Charmichael, G.L. Hug, Handbook of Photochemistry, Marcel Dekker, New York, 1993.

[23] J. Seixas de Melo, P.F. Fernandes, J. Mol. Struct. 565 (2001) 69.

[24] G. Stricker, V. Subramaniam, C.A.M. Seidel, A. Volkmer, J. Phys. Chem. B 103 (1999) 8612.

[25] A.R. Brown, N.C. Greenham, J.H. Burroughes, D.D.C. Bradley, R.H. Friend, P.L. Burn, A. Kraft, A.B. Holmes, Chem. Phys. Lett. 200 (1992) 46.

[26] D. Oelkrug, J. Gierschner, H.J. Egelhaaf, L. Luer, A. Tompert, K. Mullen, U. Stalmach, H. Meier, Synth. Met. 121 (2001) 1693.

[27] O. Narwark, S.C.J. Meskers, R. Peetz, E. Thorn-Csanyi, H. Bassler, Chem. Phys. 294 (2003) 1.

[28] O. Narwark, A. Gerhard, S.C.J. Meskers, S. Brocke, E. ThornCsanyi, H. Bassler, Chem. Phys. 294 (2003) 17.

[29] J. Seixas de Melo, F. Elisei, R.S. Becker, J. Chem. Phys. 117 (2002) 4428

[30] A. Pogantsch, A. Kai Mahler, G. Hayn, R. Saf, F. Stelzer, E.J.W. List, J.-L. Bredas, E. Zojer, Chem. Phys. 297 (2004) 143.

[31] E. Peeters, A.M. Ramos, S.C.J. Meskers, R.A.J. Janssen, J. Chem. Phys. 112 (2000) 9445.

[32] A.P. Monkman, H.D. Burrows, I. Hamblett, S. Navarathnam, M. Svensson, M.R. Andersson, J. Chem. Phys. 115 (2001) 9046.

[33] J. Grimme, M. Kreyenschmidt, F. Uckert, K. Müllen, U. Scherf, Adv. Mater. 7 (1995) 292

[34] D. Grebner, M. Helbig, S. Rentsch, J. Phys. Chem. 99 (1995) 16991.

[35] A.P. Monkman, H.D. Burrows, L.J. Hartwell, L.E. Horsburgh, I. Hamblett, S. Navaratnam, Phys. Rev. Lett. 86 (2001) 1358. 